\title{
Research on Black Silicon Based on Surface Photo Voltage
}

\author{
Baoping Kuang ${ }^{1}$, Lihua Miao ${ }^{1}$ and Tao Wang $^{2}$ \\ ${ }^{1}$ Department of Mathematics \& Physics, Shenyang Medical College, Shenyang, \\ China \\ 2 Jinzhou Power Supply Branch, State Grid Liaoning Electric Power Supply Co.
} Ltd, Jinzhou, China kbpzyky@163.com

\begin{abstract}
The solar cell research has experienced more than half a century The black silicon solar cells have the advantages of low cost, low reflectivity. Due to the bery large surface state density of Black silicon solar cells, the surface passivation effect directly affects its efficiency. Since the Surface photo voltage technolog. is very sensitive to surface states, three kinds of black silicon solar cells: no surface passivation and $\mathrm{SiO}_{2}$ passivation were tested and analyzed by this technology. The electrical characteristics of the solar cells were tested. The passivation effects were sudjed using surface photo voltage technology. We found that the recombination in the butk and on the surface dominated at shorter times and at longer times respectively from the transient photo voltage results. Surface photo voltage can reveal the generation and recombination mechanisms of light-induce charge carriers, it provides the theoretical guidance to improve the efficiency of the solar cells.
\end{abstract}

Keywords: black silicon solar Kelt, surface photo voltage technology, surface passivation, surface state'

\section{Introduction}

After entering the modern human civilization, in all aspects of science and technology, agriculture, health and culture we have made incredible achievements. While humans have been in the pursuit of higher living standards, the demand for all kinds of material resources is growing, but also the energy crisis will push the human to a dangerous situation. Curently, $85 \%$ of energy consumption comes from non-renewable coal, gas and oil and other fossil fuels, and energy has been approaching these exhausted end. At the sane time large-scale use of fossil fuels on the ecological environment has produced a variety of serious harm, for example, the greenhouse effect, acid rain, fog and haze. Human must develop new sustainable clean energy. One of the most developments potential and inexhaustible is solar - solar cells.

Monocrystalline silicon-based technology is the most mature one, but the conversion efficiency is lower. In order to further improve the conversion efficiency of crystalline silicon solar cells, researchers find ways to reduce the reflectivity of the silicon surface, the general practice is to make the surface with the pyramid structure, then to grow antireflection film. In recent years there has been a kind of silicon material-black silicon with a very low reflectivity, study the material more than ten years of history. Compared with conventional silicon material, black silicon material has significant broadband antireflection performance, black silicon visible. Near-infrared and silicon band gap less light bands have a higher absorption coefficient [1]. Strictly speaking, black silicon is not a new material, but a kind of microstructure silicon with surface modification. Preparation of black silicon surface micro-structure there are many methods, including: 
electrochemical etching method $[2,3]$, reactive ion etching method $[4,5]$, femtosecond laser pulse method [6,7], metal assisted etching, etc. [8]. Black silicon microstructure surface also take many forms, such as microporous, nanowires, microtips and so all.

Limit solar cell conversion efficiency of factors including the following processes [9]: a light-absorbing material, free carriers generated, photo-generated charge transfer to the respective electrode and from the electrode outflow. Therefore, in order to improve the photoelectric conversion efficiency of solar cells, beside the optimization mechanism of preparation process, it also need to reduce the reflection loss of incident light to enhance the effective absorption of light. Black silicon materials have good antireflection properties, making it possible to produce a material of high efficiency solar cells. However, the surface of the silicon microstructure processing Ahas been greatly increased the surface area, the consequent increase in the surface recombination also limits the efficiency. So far, the black silicon solar cell efficiency is only $18.2 \%$ [10].

Photovoltaic effect refers to the semiconductor surface, which is the result of photo-generated charge transfer and redistribution. But in practice it is not limited to the surface characteristics. SPV as a spectroscopic teempique has the following characteristics: 1) Using non-contact test, the test will not contaminate the sample and it will not damage the sample topography. At the ame time, the SPY can also detect the opaque samples which absorption spectrum (transmission spectrun) cannot do. 2) SPV information detected mainly reflects the nature of the sample surface, not far from the influence of the substrate surface. This is etearly the nature of the research of which the interface surface of the light-generated charge of the migration process is very important. 3) Since the SPV works by detectingechanges of sufface voltage caused by the incident, and therefore have a high sensitivity. Based on these advantages, in recent years, the surface of photovoltaic technolpgy has been widely used to study the photoelectric functional materials and devices. Currentlyethere are three main forms of photovoltaic technology: Kelvin probe surface selan spectrum, modulation spectrum and transient photovoltaic solar spect um. The first one is developed by Kelvin probe technology; the latter method is used by MIS. The SPV performed very well in charges behavioral mechanisms of semiconductor materials and devices, just to take advantage of its research of solar energy materials, structures and devices in the photo-generated charge characteristics, which Amproves the efficiency of solar cells and provides the basic theoretical support.

In order to increase the efficiency of black silicon solar cell, the surface must be effectively passiyity. In this chapter, we choose the simplest way - assisted etching metal prepared black silicon material. The efficiency of passivation cell has been greatly improved and-comparative analysis of surface recombination is studied by photovoltaic technology surface passivation effect.

\section{Experiment}

Black silicon surface preparation is the double structure of pyramid/nanowires. Preparation of the pyramid structure is achieved on good P-type (100) silicon substrate directly provided by the factory, a size of $125 \times 125 \mathrm{~cm}^{2}$, a resistivity of $1.3 \Omega \mathrm{cm}^{-1}$ and a thickness of $200 \mu \mathrm{m}$. Assisted etching method uses metal. The black silicon material was prepared in two steps.

Solution I: $0.01 \mathrm{M} / \mathrm{LAgNO}_{3}+4 \% \mathrm{HF}$

Solution II: $4 \% \mathrm{HF}+1.2 \% \mathrm{H}_{2} \mathrm{O}_{2}$

The concentration ratio of solution is the volume ratio. At room temperature, the first pre-cleaned silicon pyramid I, $90 \mathrm{~s}$ into the solution to deposit the silver nanoparticles. Then removed and put into solution II for silicon nanowires, the etching time was $15 \mathrm{~s}$. SEM micrographs of good black silicon prepared were carried out the reflectance tests. 
A, B represents two cells which without any surface passivation, and black silicon solar cells of $\mathrm{SiO}_{2}$ passivation respectively. For A cell, pn junction is formed in the diffusion and battery electrodes are directly prepared; for $\mathrm{B}$ cell, after diffusion, thermal oxidation layer $\mathrm{SiO}_{2}$ is prepared, finally the electrodes was prepared. Specific production process is as follows:

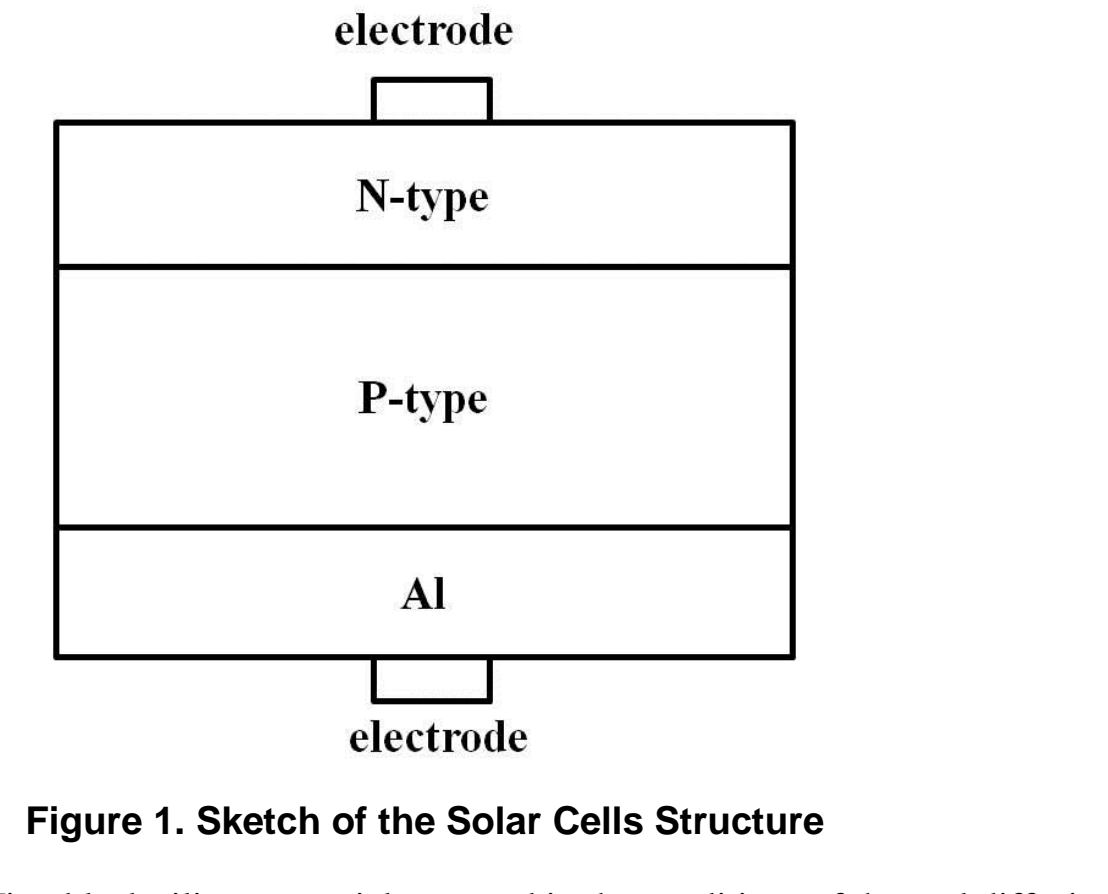

1) Diffusion: First black silicon material prepared in the conditions of thermal diffusion $500 \mathrm{~s}$ with $\mathrm{N}_{2}: \mathrm{O}_{2}=10$ : $1(1 \mathrm{w}$ w rate rato to generate oxide layer to inhibit the diffusion process subsequent diffusion of phosphorus into the body too quickly. Using phosphorus oxychloride $\mathrm{POCl}_{3}$ diffursion source, first through phosphorus diffusion source at $800^{\circ} \mathrm{C}$ $1000 \mathrm{~S}$, then the phosphorus source dvas stopped, the temperature was increased to $850^{\circ} \mathrm{C}$ continue diffusion time 600-S. After diffusion, wash the surface with HF PSG.

2) Thermal oxidation $\mathrm{in} \mathrm{N}_{2}: \mathrm{O}_{2}=1: 6$ under the condition of thermal oxidation $1500 \mathrm{~s}$, a temperature of $800^{\circ} \mathrm{C}$

3) Preparing thereleetrodes: made for each electrode by screen printing and sintering prepared.

\section{Results and Discussion}

\section{Characterization of Black Silicon}

Figure 2 shows the surface of black silicon. The SEM photograph is produced by HITACHI S-4800 field emission scanning electron microscope. Tilted 30 degrees from the surface of the figure can be seen as the entire black silicon surface, like wooded mountains, but also in each side of the pyramid are very evenly distributed nanowires, indicating metal assisted etching in various crystallographic orientation. As can be seen from a cross-sectional view, the length of the nanowire is about $200 \mathrm{~nm}$, a diameter is about 70-200 $\mathrm{nm}$. The whole pyramid nanowires are more uniform size and distribution. Figure 3 shows the reflectance values of black silicon surface within the range of $300-1100 \mathrm{~nm}$, in this wavelength range, the average reflectivity is $6.49 \%$. But usually the average reflectance with only the pyramid structure of single-crystal silicon antireflective surface is greater than $15 \%$ [11]. Visible reflectance of black silicon material prepared in this letter performs a more excellent value. Experiments also noted the increase over time in the etching solution II, the length of the nanowire is also increasing, and will continue 
to reduce the reflectivity, or even below $2 \%$. However, when using of screen printing to prepare electrodes, the prepared electrodes are particularly easily fell off if the nanowires are longer than $200 \mathrm{~nm}$. Another major research aim of this letter is to use the influence of surface photovoltaic spectroscopy to analysis the cell efficiency after surface passivation, which chooses black silicon material preparation of lower reflectivity with much practical significance. Therefore, in order to facilitate the preparation of the electrodes, the nanowire length of less $200 \mathrm{~nm}$ is chosen to produce batteries.

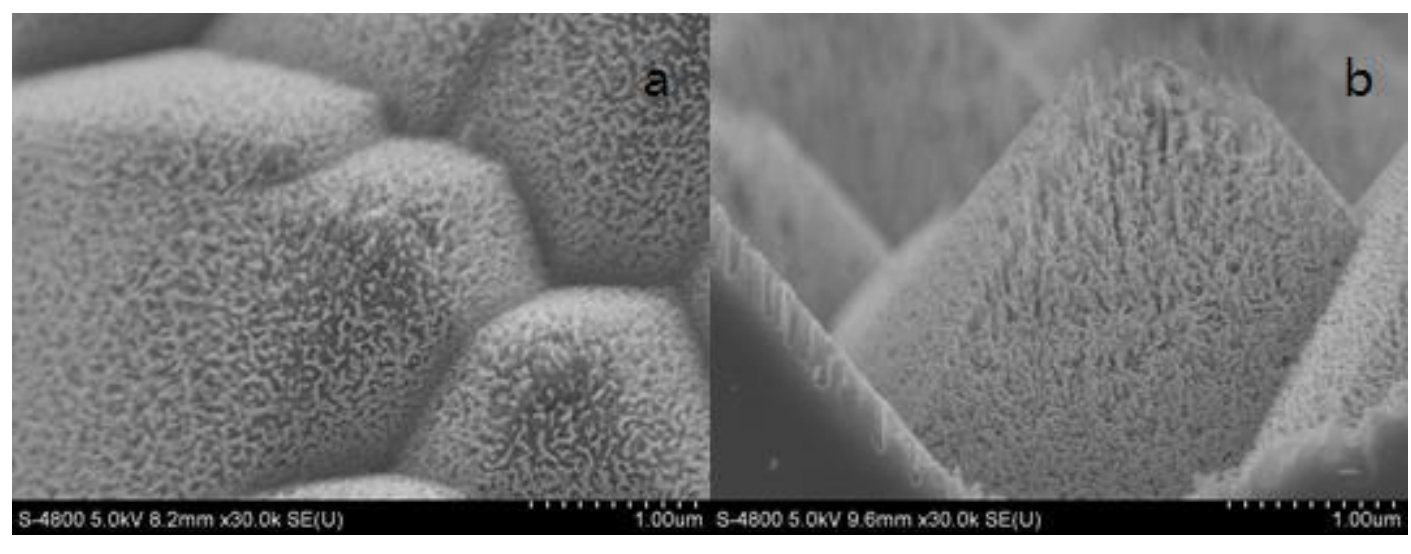

Figure 2. SEM Images OI Black Silicon Surface

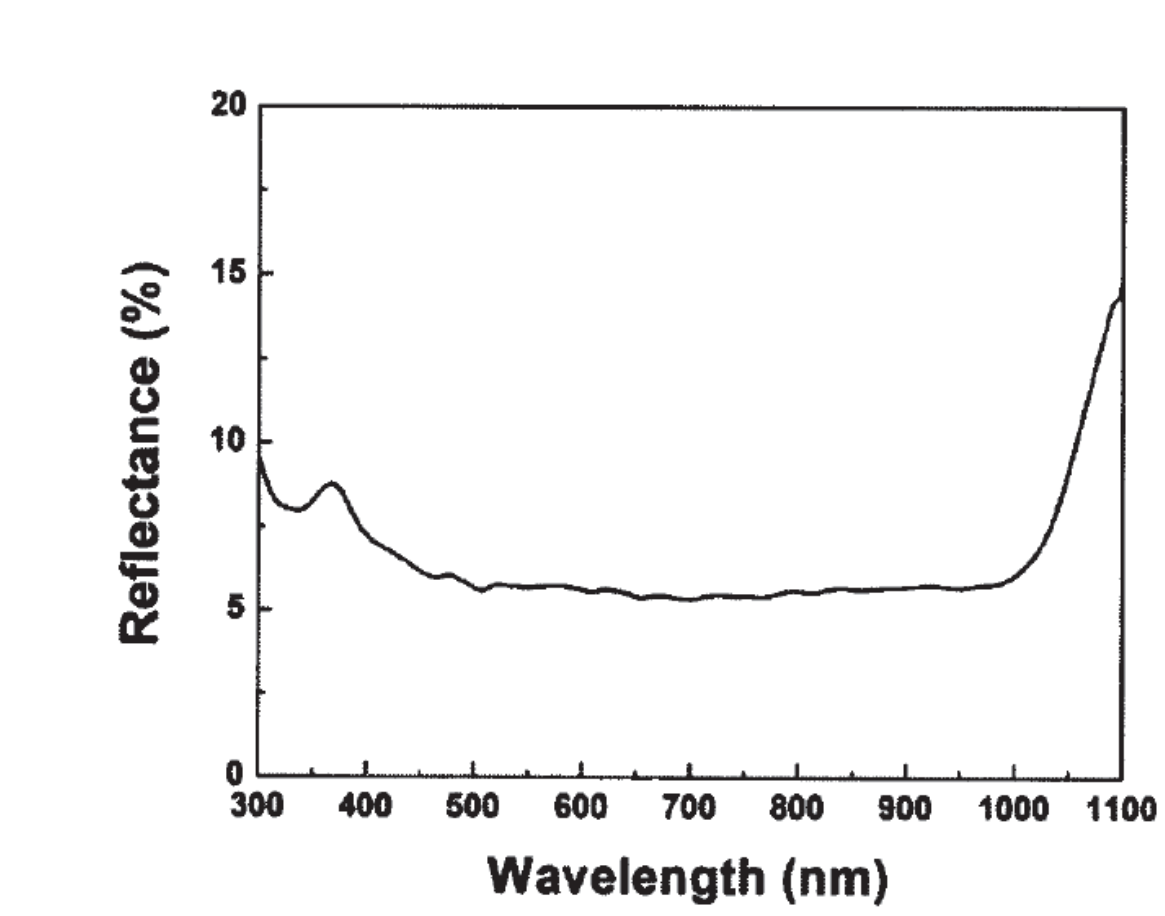

Figure 3. Reflectance of Black Silicon Surface

The formation of nanowires etching process is naturally formed under silver catalysis, catalytic etching reaction can be divided into the following two sections:

Cathode reaction:

$\mathrm{Ag}^{+}+\mathrm{e}^{-} \rightarrow \mathrm{Ag}^{0}$

Anode reaction:

$\mathrm{Si}+2 \mathrm{H}_{2} \mathrm{O} \rightarrow \mathrm{SiO}_{2}+4 \mathrm{H}^{+}+4 \mathrm{e}^{-}$

$\mathrm{SiO}_{2}+6 \mathrm{HF} \rightarrow \mathrm{H}_{2} \mathrm{SiF}_{6}+2 \mathrm{H}_{2} \mathrm{O}$ 


\subsection{Electrical Properties of Black Silicon Solar Cells}

First, two cells were tested electrical characteristics, and Table 1 gives the electrical parameters. Seen from the table, after passivation, the electrical parameters of the battery are improved, photoelectric conversion efficiency of the battery increase from $11.8 \%$ to $16.3 \%$. Because the diffusion and electrode preparation and other processes are the same, it is reasonable to identify that the ways to improve battery performance is caused by the surface passivation.

Table 1. The Parameters of Black Silicon Solar Cells

\begin{tabular}{|l|l|l|l|l|}
\hline & $\mathrm{V}(\mathrm{v})$ & $\mathrm{I}(\mathrm{A})$ & $\mathrm{FF}(\%)$ & $1(\%)$ \\
\hline $\mathrm{A}$ & 0.58 & 7.22 & 64.63 \\
\hline $\mathrm{B}$ & 0.62 & 8.32 & 16.11
\end{tabular}

Theoretically, the battery output power loss relates with the optical absorption and carrier recombination processes. Junction diffusion effects and electron-hole recombination limits the battery fill factor AF. First of all, If the diffusion effect of batteries $\mathrm{A}$ and $\mathrm{B}$ is the same, then the fill factor of the battery $\mathrm{A}$ is limited by recombination of electron-hole. FF value determines the position of the maximum output power point. Series resistance and parallel resistance have an impact on the FF. The series resistor R plays a major role in feducing thefinl factor FF, especially for large-scale commercial batteries.

For a given type of battery, smaller series Tesistance and greater resistance join together to give a higher cell efficiency [12]. Low surface recombination velocity helps to improve the short-circuit currênt, thus improving the cell efficiency.

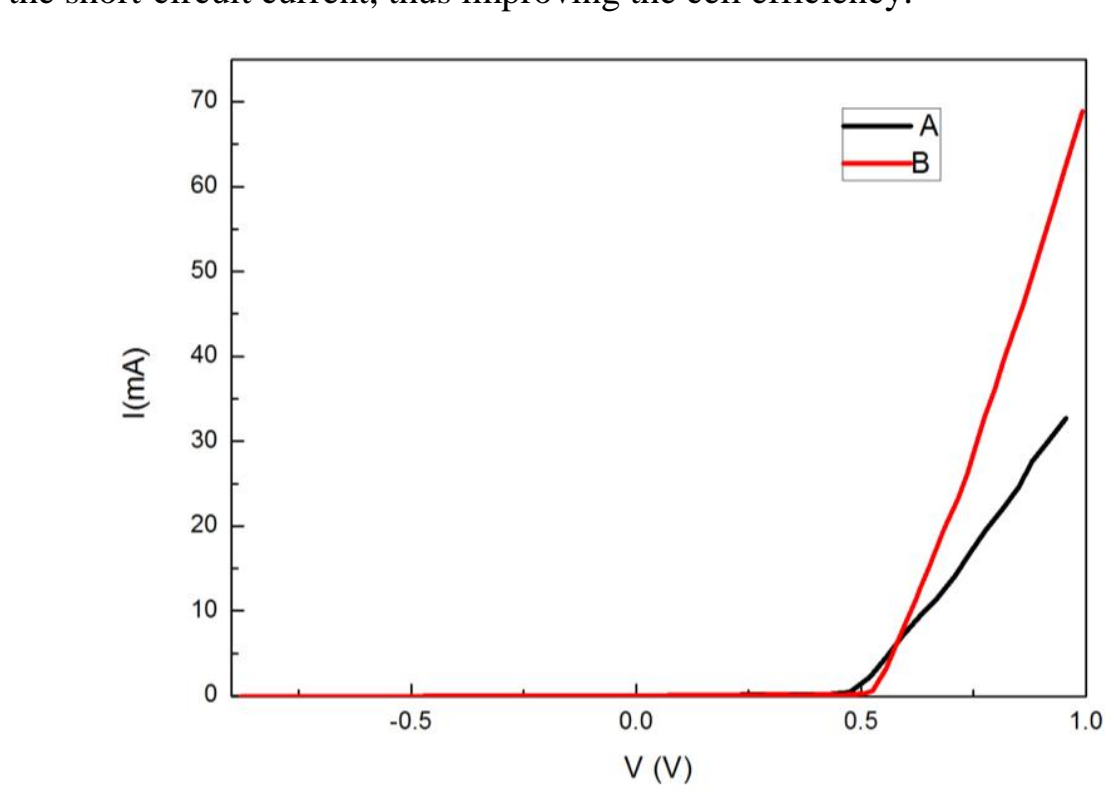

Figure 4. Dark I-V Characteristics of Solar Cells A and B

In addition, two cells were also tested IV characteristics in a dark state, as shown in Figure 4. Under a large forward bias conduction, series resistance determines the size of current. The greater of the current, the smaller of the series resistance. While in the 
reverse bias, the diode is turned off, the current size of the cell is determined by parallel resistance, the greater of the current, and the smaller of shunt resistance. Thus, the series resistance of batteries A, B is gradually reduced, while the parallel resistance R increases, which consistent with the results in Table 1 . Because in addition to the passivated silicon surface of two different battery, and other manufacturing processes are the same, so that black silicon surface passivation caused the reduced resistance, the smaller series resistor means that a smaller contact resistance, and the larger of shunt resistance (reverse current smaller) means that leakage current of black silicon surface is smaller. Based on the above analysis, after surface passivation, the contact resistance between the metal electrode and the battery B is reduced, and the surface defect density significantly reduced, which reduces the surface recombination velocity.

\subsection{Black Silicon Photovoltaic Solar Cells Modulation Spectrum}

To study the effect $\mathrm{SiO}_{2}$ passivation of the battery characteristics, the lock in amplifier modulation spectrum of photovoltaic cells were characterized by measurements in air at room temperature, the monochromatic light incident from black silicon surface.

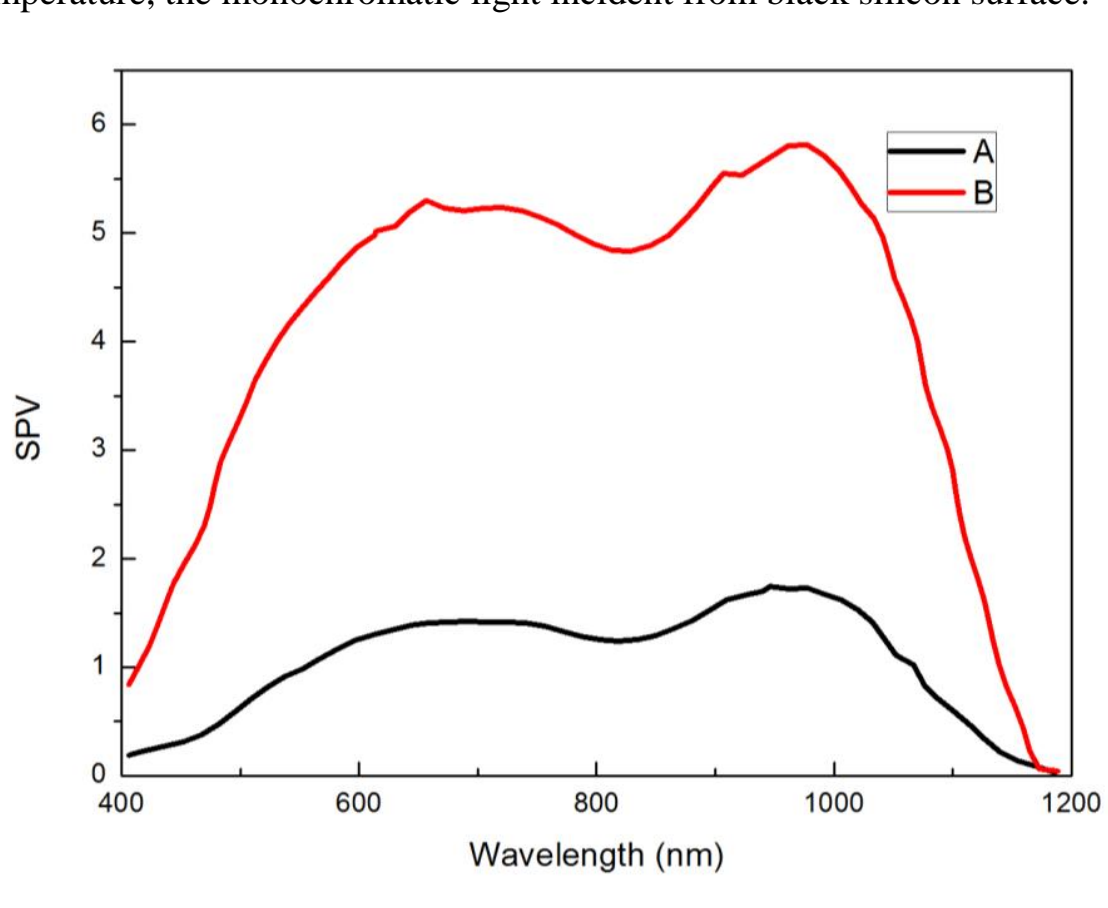

Figure 5. Modulated SPV Spectra of Black Silicon Solar Cells A and B

SPV can be seen from Figure 5. The surface with non-passivated of battery A in the $400-1200 \mathrm{~nm}$ wavelength range is response to the minimum, and the photovoltaic response of battery B through the surface passivation greatly improved. Parameter of P-type silicon substrate and the diffusion process of the two batteries are same, so that the internal electric field of the pn junction is same, which means that the built-in electric field has the same capacity to separate photogenerated electron-hole pairs. So in the same light intensity, if the life of the carriers is long, the corresponding SPV response becomes large.

In general, the emitter diffusion in silicon cell expression of minority carrier effective life:

$1 / \tau_{\text {eff }}=1 / \tau_{\text {bulk }}+\left(S_{\text {eff }}^{F}+S_{\text {eff }}^{B}\right) / 1$

$\tau_{\text {eff }}$ is the life of Shockley-Read-Hall, $S_{\text {eff }}^{F}$ and $S_{\text {eff }}^{B}$ are front and rear surfaces rate of the effective surface recombination, $d$ is the thickness of the silicon wafer. Due to 
the same parameters of silicon, a back electrode process is also the same, it is assumed that two batteries of $\tau_{\text {bulk }}$ and $S_{\text {eff }}^{B}$ are the same, so the impact on $\tau_{\text {eff }}$ is negligible, then the only factor affecting the minority carrier lifetime of the battery is $S_{\text {eff }}^{F}$. From the above formula, the front surface of the effective recombination velocity $S_{\text {eff }}^{F}$ is smaller, the battery effective minority carrier lifetime $\tau_{\text {eff }}$ is greater, while increasing the minority carrier lifetime will increase the photovoltaic cell response amplitude spectrum. Based on the above analysis, we can draw the larger photovoltaic cell response will give a smaller effective rate of the front surface. In conjunction with Figure 5, black silicon surface recombination rate of B sequentially reduced. Visible, modulated spectrum analyze the surface of the photovoltaic cell surface passivation is concerned, although not quantitatively give the exact value of the surface recpmbination velocity directly, but can be qualitatively analyzed passivation effect.

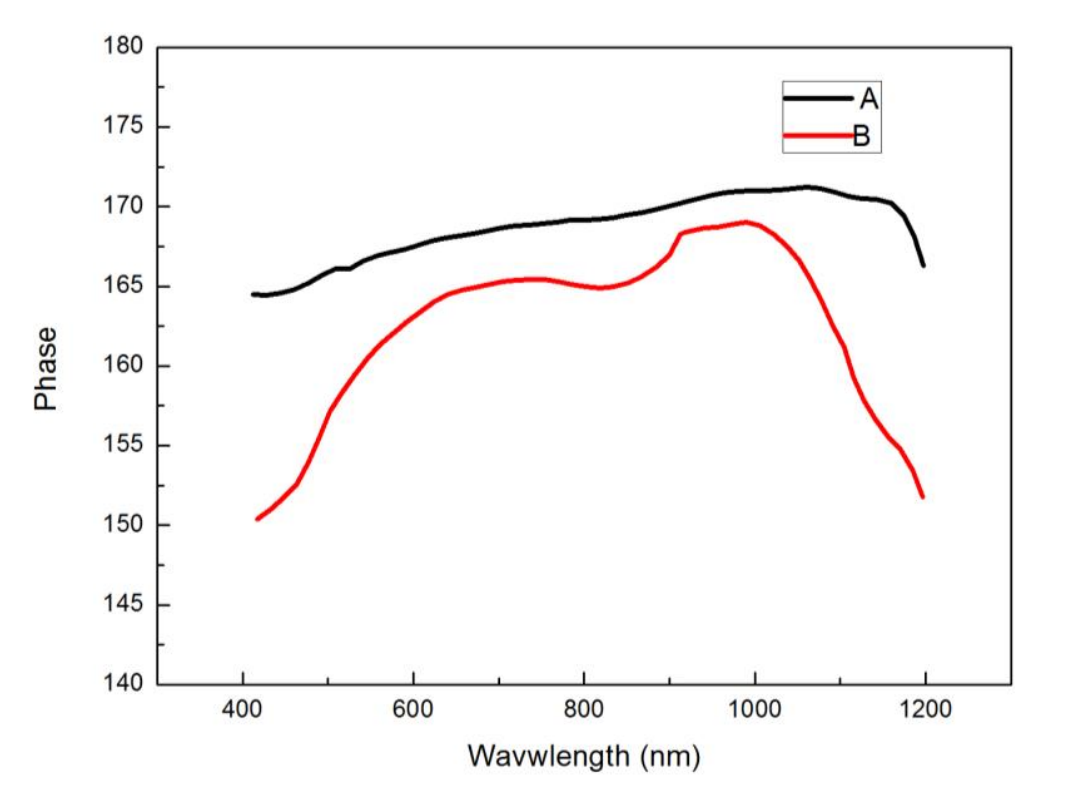

Figure 6. Phase Spectra of Black Silicon Solar Cells A and B

Due to the presence of a strong electric field within a pn junction cell, the photo-generated electrons bound to move toward the surface of n-type diffusion region, photo-generated holes move towards to the direction of back electrode. It can also be seen from the phase diagram of Figure 6, in 1200-1000 nm band, the absorption coefficient of silicon is increasing, the phase values of two cells are shifted to 180 degrees, which indicated that after the illumination light, generated electrons move toward the surface, the hole move towards to the backside electrode, and this is expected to result from the uniform cell structure. It can also be seen from the Figure that the degree of the phase value of the battery A, B of the deviation from 180 degrees is gradually increased. This is because excess carrier lifetime of all two cell in turn increases within a given exposure time, the time to reach a stable equilibrium value needed by instantaneous excess carrier concentration will also increase. In terms of a single cell in the 1200-1000 nm wavelength range, the absorption coefficient of the material increases, resulting in a transient increase in the excess carrier concentration, reducing the instantaneous carrier lifetime, so that the given exposure time is reduced, excess carrier concentration can reach a stable equilibrium value in a shorter period of time, corresponding to the phase angle of 180 degrees with respect to the extent of the delay is smaller. The surface recombination velocity of Battery A is too large, the impact of increasing the absorption coefficient of 
the instantaneous excess carrier lifetime is reduced, change the value of the phase angle is relatively reduced. The value of the phase angle of $600-400 \mathrm{~nm}$ increases, the light intensity is reduced by the excess carrier concentration leads to decrease.

\subsection{Black Silicon Photovoltaic Solar Cells Transient Spectroscopy}

Firstly, the system test the change of that Kelvin probe open or close $1000 \mathrm{~nm}$ monochromatic light battery CPD with time, the results are shown in Figure 7. When the test is carried out, the back of the battery electrode is grounded, the CPD value change of black silicon side is tested, and the probe is placed between the two adjacent metal gate line electrode. Since the surface of the black silicon battery B is grown $\mathrm{SiO}_{2}$ passivation layer,the surface work function of two cell is not necessarily the same under a black state. It can be seen from Figure 7, CPD value in black state of battery A, B and the reference electrode is $160 \mathrm{mV}$ and $240 \mathrm{mV}$ respectively, which is due to the passivation to make the cell surface band bending decrease. The pn junction of battery A and B is the same, the only difference is the passivation layer. Since $\mathrm{SiO}_{2}$ passivation layer døes notabsorb 1000 $\mathrm{nm}$ monochromatic to produce free charge, the battery $\mathrm{A}$ and $\mathrm{B}$ absolb light generating mechanism of free charge should be the same. Wherr the $1000 \mathrm{~nm}$ light turned on, CPD change of the battery A is $30 \mathrm{mV}$, which amount of the battery $B$ is $120 \mathrm{mV}$. This shows that after the light turning on, the number of negatively charged aggregate surfaces on the batteries A, B sequentially increased. After passivation, the surface recombination rate of black silicon is significantly reduced, which is consistent with the previous analysis.

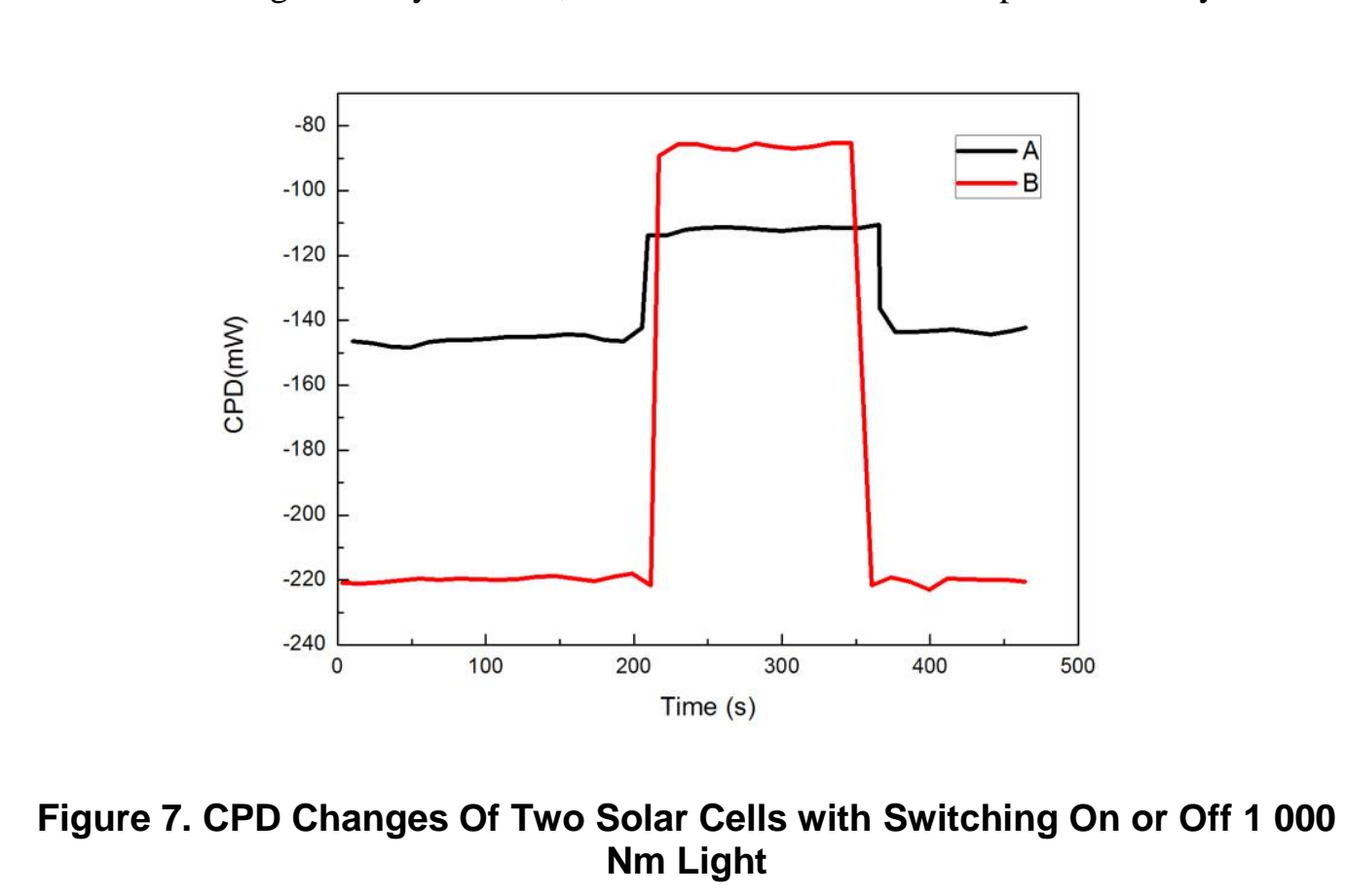

\section{Conclusion}

In this letter, the black silicon of a large pyramid / nanowire double structure is prepared by metal assisted etching method. The Black silicon surface has a nano-uniform structure and low reflectivity. Then, two black silicon solar cells of non-surface passivation and surface passivation by $\mathrm{SiO}_{2}$ are prepared. Firstly, both the electrical parameters of two batteries are tested, and the characteristics of the passivation one have been increased dramatically. Secondly, the passivation effect of two cells is discussed in detail by modulation photovoltaic spectrum, and the good passivated surface is responsible for increased photovoltaic response. Finally, body recombination plays a 
leading role in transient photovoltaic attenuation on a short time by two photovoltaic cell transient spectroscopy, and surface recombination plays a leading role on a long period of time.

\section{References}

[1] L. Escoubas, R. Bouffaron and V. Brissonneau, "Sand - castle biperiodic pattern for spectral and angular broadening of antireflective properties", Optics letters, vol. 35, no. 1455, (2010).

[2] L. L. Ma, Y. C. Zhou and N. Jiang, "Wide-band "black silicon" based on porous silicon", Appl. Phys. Lett, vol. 88, no. 171907, (2006).

[3] R. R. Bilyalov, L. Stalmans and L. Schirone, "Use of porous silicon antireflection coating in multicrystalline silicon solar cell processing", Electron Devices. IEEE Transactions on, vol. 46, no.

2035, (1999).
[4] T. P. Chow, P. A. Maciel and G. M. Fanelli, "Reactive Ion Etching of Silicon in $\mathrm{CCl}_{4}$ and $\mathrm{HCI}$ Plasmas", J. Electrochem. Soc., vol. 134, no. 1281, (1987).

[5] H. Jansen, N. Gardeniers and M. de Boer, "A survey on the reactive ion etching of silicon in microtechnology", Journal of micromechanics and microengineering, vol. 6, no. 14, (1999).

[6] T. H. Her, R. J. Finlay and C. Wu, "Microstructuring of silicon with femtosecønd laser pulses", Appl. Phys. Lett., vol. 73, no. 1673, (1998).

[7] R. Le Harzic, H. Schuck and D. Sauer, "Sub100 nm nanostucturing of silicon by ultrashort laser pulses", Optics Express, vol. 13, no. 6651, (2005).

[8] S. Koynov, M. S. Brandt and M. Stutzmann, "Black nonreflecting silicon surfaces for solar cells", Appl. Phys. Lett., vol. 88, no. 203107, (2006).

[9] S. R. Forrest, "The limits to organic photovoltaic eell efficiency", MRS Bulletin, vol. 30, no. 28, (2005).

[10] J. Oh, H. C. Yuan and H. M. Branz, "An 18.2\%-efficient black-silicon solar cell achieved through control of carrier recombination in nanostructures", Nature Nanotechnology, vol. 7, no. 743, (2012).

[11] H. H. Li, W. F. Liu and A. M. Liu, "Metal grids - based texturization of monocrystalline silicon wafers for solar cells", Sol. Energy Mater. Sol Cells, vol. 94, no. 942, (2010).

[12] U. Stutenbaeumer and B. Mesfin, sEquivalent Model of monocrystalline, polycrystalline and amorphous silicon solar cells", Renewable energy, vol. 18, no. 501, (1999).

\section{Nathors}

Baoping Kuang, He is professorial senior experimenter and director of the physics laboratory of Shenyang Medical College, also director of Liaoning Institute of physics, director of Liaoning institute of cognitive and behavioral sciences, and researcher of Liaoning Provincial Key Laboratory of behavioral and cognitive neuroscience. He graduated from Bohai University, majoring in theoretical physics, and is mainly engaged in physics, electronics research and teaching, behavior and cognitive neuroscience research, and animal behavior training instrument $\mathrm{R} \& \mathrm{D}$ and application. He won the second prize of Liaoning province science and technology progress award once, and second prize of Shenyang science and technology progress award once as well, with 6 authorized national invention patents and 6 utility model patents.

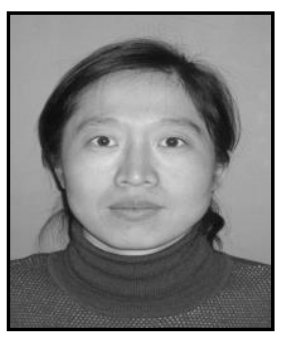

Lihua Miao, She received the Master degree in Physics from Shenyang Normal University, Shenyang, China, in 2006.

She is currently an associate professor with the Department of Computer and Mathematics \& Physics, Shenyang Medical College, Shenyang. Her current research interests include chaotic control in medical field and neural networks theory. 
International Journal of Control and Automation

Vol. 9, No. 8 (2016)

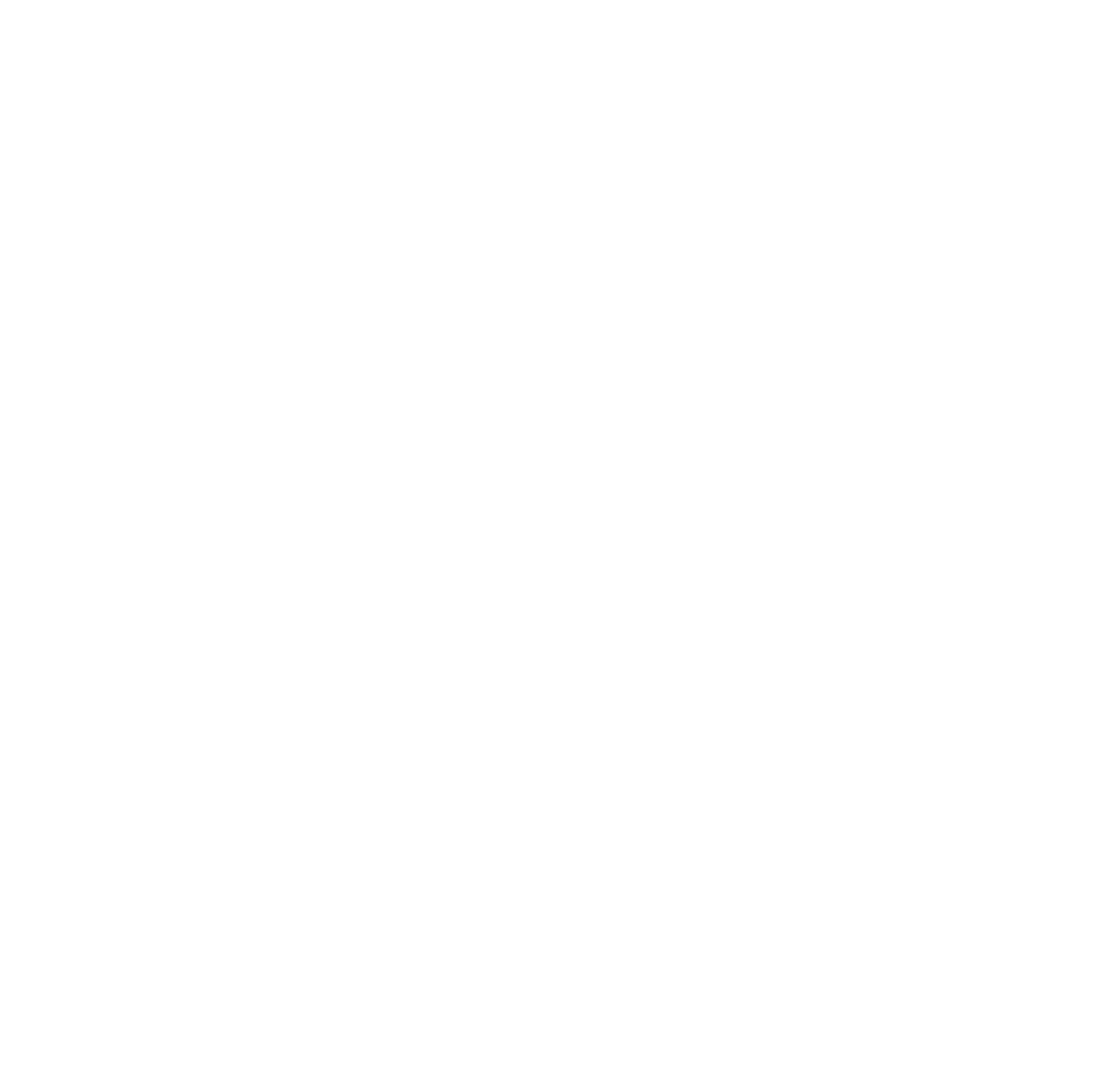

